\title{
Abrasion injuries on artificial turf: A real risk or not?
}

\author{
D M Twomey, ${ }^{1} \mathrm{PhD} ; \mathrm{L}$ A Petrass, ${ }^{1} \mathrm{PhD} ; \mathrm{P}$ R Fleming, ${ }^{2} \mathrm{PhD}$ \\ ${ }^{1}$ Faculty of Health, Federation University Australia, Mt Helen Campus, Victoria, Australia \\ ${ }^{2}$ School of Civil and Building Engineering, Loughborough University, United Kingdom
}

Corresponding author: D M Twomey (d.twomey@federation.edu.au)

\begin{abstract}
Abrasion injuries result in damage only to the surface layer of skin and can result in player discomfort and changes in performance. The perceived fear of abrasion injuries on artificial turf playing surfaces has significantly affected the adoption of these surfaces, particularly in sports that involve frequent player-surface interactions. The underreporting of abrasion injuries due to how time-loss injuries are defined and the lack of validity of the current abrasion measurement device highlight the need for more research to understand fully the incidence and nature of abrasions on artificial turf playing surfaces and the effect of these injuries on playing behaviour. Improved reporting of abrasion injuries and a more biofidelic test device could assist in both the development of abrasion-related injury prevention strategies and in dispelling players' negative perceptions of abrasions on artificial turf.
\end{abstract}

S Afr J SM 2014;26(3):91-92. DOI:10.7196/SAJSM.563

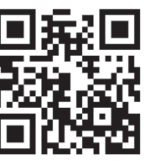

Abrasion injuries result in damage only to the surface layer of skin (epidermis), and while typically classified as minor in nature, can engender player discomfort and consequently result in a change in playing behaviour. ${ }^{[1]}$ Strategies to reduce the incidence of abrasion injuries in sport are therefore desirable. While it is difficult to replicate natural turf fully, the perceived fear of abrasion-type injuries on artificial turf is surprising given the fact that, unlike natural turf, artificial turf fields are required to satisfy a set of safety and performance standards that include an abrasion measure.

Injury studies on the earlier generations of artificial turf products (i.e. prior to the late 1990s) consistently reported an increase in the number of abrasion injuries on artificial turf compared with natural grass. ${ }^{[2]}$ Since then, studies have been undertaken on third-generation $(3 \mathrm{G})$ surfaces with longer fibres and infilled with rubber and sand, but abrasion injuries are still consistently higher than on natural turf. In terms of total injuries recorded in published injury studies, however, overall abrasion injury rates are relatively low, ranging from $\sim 2.5 \%$ to $6 \%{ }^{[3,4]}$ These low rates may be largely owing to the injury definitions used, which are generally based on time loss and may be a rare occurrence with abrasion-type injuries unless they are recurrent or become inflamed/infected. More detailed and accurate reporting of abrasion injuries in future injury studies is needed to appreciate the extent of the problem fully.

Another issue that arises when interpreting abrasion injury incidence to date is that this type of injury is regularly reported with lacerations (injuries to multiple layers of skin) or as part of skinrelated injuries; therefore, it is difficult to have confidence in the relative risk of abrasion injuries alone. Given that the mechanism of laceration injuries is very different to abrasion injuries, strategies to prevent these injuries need different considerations. Consequently, it is plausible that the total incidence of abrasion injuries may be significantly underestimated in many injury epidemiological studies, and the effect of these injuries could be much greater than anticipated.

Recently, Peppelman et al ${ }^{[5]}$ were the first to attempt to increase understanding of the effect of sliding on natural and $3 \mathrm{G}$ artificial turf surfaces on in vivo human skin. They concluded that sliding on natural grass resulted in more erythema (redness), but fewer abrasions compared with sliding on artificial turf. They contended that these observed differences may be important in studying the skin comfort of players using artificial turf surfaces, and suggested that the discomfort associated with abrasion injuries can negatively influence players' performance and change their playing behaviour. ${ }^{[5]}$ If players are changing their playing behaviour to protect skin abrasions from further injury during the regeneration and remodelling phases of healing, they may in fact increase their risk of other injuries. More detailed and accurate reporting in this area would provide valuable insights into the real effect of abrasion injuries.

Regardless of the lack of epidemiological data on abrasion injuries on $3 \mathrm{G}$ artificial turf, it appears that players perceive abrasion injuries as a real risk and a potential barrier to their acceptance of such surfaces. Due to the early adoption of $3 \mathrm{G}$ artificial turf in soccer, player perceptions of $3 \mathrm{G}$ artificial turf are limited predominantly to this sport. Consistently, players have concerns about the effect of the surface on both technical and physical performance, particularly the risk and severity of abrasions as a result of sliding tackles and falls on artificial turf. ${ }^{[6-8]}$

In the Italian Amateurs League, over 1600 male players aged between 15 and 35 years reported concerns about the risk of abrasion in sliding tackles. ${ }^{[8]}$ In terms of abrasions, players' role in the team (goalkeeper, defender, midfielder and forward) influenced their perception, with defenders and midfielders scoring the surface worse for abrasions than goalkeepers and forwards. This finding is 
likely to be explained by the playing characteristics associated with these different roles, with defenders and midfielders more likely to have recurrent player-ground interaction due to sliding tackles and attempting intercepts. In a more recent study, Spanish male amateur football players (aged 16 - 36 years) provided very poor ratings for the safety aspects associated with artificial turf, including skin abrasions, muscle strains and the general possibility of sustaining an injury. ${ }^{[6]}$ For most players, the biggest disadvantage associated with artificial turf was skin abrasion, specifically in sliding tackles. Perceptions of amateur-level players are consistent with studies conducted with elitelevel players. A large study conducted with male $(n=1018)$ and female $(n=111)$ elite players of similar age to participants in the previous studies indicated that $>60 \%$ felt that artificial turf was too abrasive. ${ }^{[7]}$ While artificial turf provides a valuable alternative to natural grass in areas experiencing extreme climatic conditions, negative player perceptions need to be addressed to ensure that these surfaces are successfully adopted.

Artificial surfaces are becoming increasingly popular for a wide range of other football codes, especially with surfaces designed for multisport usage. As abrasion has been identified as an issue for soccer, this is likely to emerge as a more prominent issue for players of other football codes that have greater player-surface contact through various forms of sliding tackles. In fact, the perceived risk of abrasion and discomfort may start to affect players' uptake of these surfaces for both training and competition, which could negatively affect participation rates and the associated health benefits. While there is huge variability and substantial product development and improvement in artificial turf, which could change the properties of these surfaces, it is still possible that users' perceptions are likely to be largely influenced by available information and the appearance of the facility. It is important that the abrasion measures performed on all emerging artificial turf products are ecologically valid to accurately reflect the level of abrasion likely to be encountered when players interact with the surface during tackling manoeuvres.

Currently, 3G artificial turf products need to satisfy an abrasion standard in order to be certified for use by governing bodies of specific sports. In the football codes of soccer, rugby union, rugby league, Australian football and Gaelic football, a mechanical device called the Securisport is approved to measure a value for skin abrasion and skin friction of the surface. This device firstly measures the coefficient of friction between a test foot (covered in a silicone skin) and a polished steel plate, and then between the test foot and the artificial turf product. The level of abrasiveness of the surface is quantified as the percentage difference between the force measures on the steel plate with the new skin compared with the abraded skin after it has been tested on the artificial turf product. A major limitation of this device is the way in which it moves over the artificial surface during testing. The test foot performs five complete revolutions at a speed of 40 (standard deviation 1) revolutions/minute under a very low normal force of $100 \mathrm{~N}$. Interestingly, there is no supporting research evidence for five revolutions representing an appropriate simulation of the level of damage or the level of normal load, and this rotational movement does not replicate the player surface interaction of many sports played on 3G artificial turf surfaces. This lack of biofidelity highlights the fact that although artificial turf may satisfy abrasion standards, some artificial turf products may indeed be too abrasive to withstand the player-surface interaction of the sport without resulting in injury to the skin.

In conclusion, players perceive an increased risk of abrasion injuries on artificial turf, which leads to a negative attitude in the adoption of these surfaces. Owing to the time-loss injury definitions used in many injury epidemiological studies, it is likely that abrasion injuries are underreported and hence their effect on players is underestimated. More work is needed to improve the reporting of abrasion injuries and validity of testing devices to understand fully the incidence and nature of abrasions on artificial turf surfaces, and the effect of these injuries on playing behaviour.

\section{References}

1. Van den Eijnde WAJ, Peppelman M, Lamers EA, et al. Understanding the acute skin injury mechanism caused by player-surface contact during soccer: A survey and systematic review. Orthopaedic Journal of Sports Medicine 2014;2(5):2325967114533482. [http://dx.doi.org/10.1177/2325967114533482]

2. Ekstrand J, Nigg B. Surface-related injuries in soccer. Sports Med 1989;8(1):56-62.

3. Soligard T, Bahr R, Andersen TE. Injury risk on artificial turf and grass in youth tournament football. Scand J Med Sci Sports 2012;22(3):356-361. [http://dx.doi. org/10.1111/j.1600-0838.2010.01174.x]

4. Fuller CW, Dick RW, Corlette J, et al. Comparison of the incidence, nature and cause of injuries sustained on grass and new generation artificial turf by male and female football players. Part 2: Training injuries. Br J Sports Med 2007;47(Suppl 1):i27-i32. [http://dx.doi.org/10.1136/bjsm.2007.037275]

5. Peppelman M, van den Eijnde WAJ, Langewouters AMG, et al. The potential of the skin as a readout system to test artificial turf systems: Clinical and immunohistological effects of a sliding on natural grass and artificial turf. Int J Sports Med 2013;34(9):783788. [http://dx.doi.org/10.1055/s-0032-1331173]

6. Burillo P, Gallardo L, Felipe JL, et al. Artificial turf surfaces: Perception of safety, sporting feature, satisfaction and preference of football users. Eur J Sport Sci 2014;14(Suppl 1):S437-S447. [http://dx.doi.org/10.1080/17461391.2012.713005]

7. Roberts J, Osei-Owusu P, Harland A, et al. Elite football players' perceptions of football turf and natural grass surface properties. Procedia Eng 2014;72:907-912. [http://dx.doi.org/0.1016/j.proeng.2014.06.150]

8. Zanetti, EM. Amateur football game on artificial turf: Players' perceptions. Appl Ergon 2009;40(3):485-490. [http://dx.doi.org/10.1016/j.apergo.2008.09.007] 